\title{
Einheit in der Inneren Medizin - erhalten und pflegen
}

„Die Deutsche Gesellschaft für Innere Medizin e.V. (DGIM) wurde 1882 in Wiesbaden als „Congress für innere Medicin“ gegründet. Sie gehört heute mit mehr als 20000 Mitgliedern zu den größten medizinisch-wissenschaftlichen Fachgesellschaften in Deutschland. Die DGIM vertritt die Anliegen der Internisten in Klinik und Praxis, in Forschung und Lehre, in Fort- und Weiterbildung. Sie ist die Interessenvertretung der Inneren Medizin in Politik und Öffentlichkeit, ebenso gegenüber Behörden und Organisationen der ärztlichen Selbstverwaltung. Als gemeinnütziger Verein fördert die Gesellschaft Wissenschaft und Forschung auf dem gesamten Gebiet der Inneren Medizin. Sie versteht es als ihren Auftrag, wissenschaftliche Erkenntnisse in angewandte Heilkunde zu übertragen und so die Fortschritte der Inneren Medizin unmittelbar dem Patienten zugute kommen zu lassen. Durch Fortbildungsangebote sichert sie den Transfer medizinischer Errungenschaften in den Alltag von Klinik und Praxis. Zu diesem Zweck setzt sie sich für die Integration der Spezialgebiete der Inneren Medizin ein und pflegt die Beziehungen zu den wissenschaftlichen Schwerpunktgesellschaften."

(www.dgim.de | Über die Gesellschaft)

Begreift man die Innere Medizin als die Summe ihrer Schwerpunkte, dann ergibt sich daraus ein Fach. Dieses repräsentiert sich jedoch nicht mehr ohne Weiteres in einer Person, also einem Facharzt, denn die ständig wachsenden Erkenntnisse aller Schwerpunkte übersteigen die Erfahrungsmöglichkeiten einer einzelnen Person. Begreift man die Innere Medizin aber als die Spezialkenntnisse darüber, welche Auswirkungen Organ- oder Systemerkrankungen auf den Organismus haben und was sie für die Entstehung, die Diagnostik, den Verlauf und die Therapie anderer Organ- oder Systemerkrankungen bedeuten, dann hat man den Internisten vielleicht am besten beschrieben. Dieser Internist kann durchaus entsprechend seinen Interessen auch subspezialisiert sein, also wie

\section{Schwerpunkte der DGIM}

- Angiologie
- Endokrinologie und Diabetologie
- Geriatrie
- Hämatologie und Onkologie
- Infektiologie
- Intensivmedizin
- Kardiologie
- Nephrologie
- Pneumologie
- Rheumatologie
- Verdauungs- und Stoffwechselkrankheiten

ich zum Beispiel Kardiologe, aber er muss sich ein breites Wissen in der Inneren Medizin erwerben, er muss es erhalten und pflegen.

Die DMW ist bereits seit 1995 Organ der DGIM, und die meisten Mitglieder werden mit ihr vertraut sein. Erstmals mit dem vorliegenden Heft wird nun die DGIM neben den bereits gewohnten Mitteilungen gemeinsam mit der DMW ganze Gesellschaftsausgaben veröffentlichen. Diese Gesellschaftsausgaben werden alle zwei Monate, also sechsmal im Jahr erscheinen. Jede dieser Ausgaben wird neben den Mitteilungen aus der DGIM die neuesten Erkenntnisse und Entwicklungen aus ihren elf Schwerpunkten (s. Kasten) darstellen.

In allen sechs Ausgaben jedes Jahres formulieren kompetente Autoren ein Update für ein Krankheitsgebiet oder Thema aus jedem der Schwerpunkte, wie zum Beispiel in dieser ersten Ausgabe das Asthma bronchiale für den Schwerpunkt Pneumologie, die koronare Herzkrankheit für die Kardiologie oder die Mangelernährung für die Geriatrie - insgesamt also 66 Beiträge pro Jahr.

Die DMW Leserinnen und Leser kennen diese Form aus den Sonderheften „Klinischer Fortschritt", die bisher in der DMW seit vielen Jahren regelmäßig im Sommer erschienen. Dabei wird keine umfängliche Übersicht über ein Thema gegeben. Stattdessen werden wichtige neue Studien, Leitlinien oder Kontroversen kurz berichtet und aus Sicht der Autoren kritisch bewertet. Ganz im Vordergrund steht die Relevanz für die klinische Praxis. Von Jahr zu Jahr werden entsprechend den Entwicklungen und neuesten Forschungsergebnissen die Themengebiete dann etwas unterschiedlich sein.

Eine wichtige Rolle spielen in diesem Konzept die Schwerpunkt-Herausgeber, denen ich an dieser Stelle ebenso wie den Autoren herzlich für ihr großes Engagement danken möchte. Sie wählen für ihren jeweiligen Schwerpunkt die Themen aus, sprechen Autoren an und sorgen gemeinsam mit der Redaktion für die Umsetzung der aktuellen Beiträge in der gewohnten hohen DMW Qualität.

Wir hoffen, dass wir unseren Mitgliedern und allen DMW Leserinnen und Lesern auf diese Weise ermöglichen, ihr breites Wissen in der Inneren Medizin zu erhalten und zu pflegen.

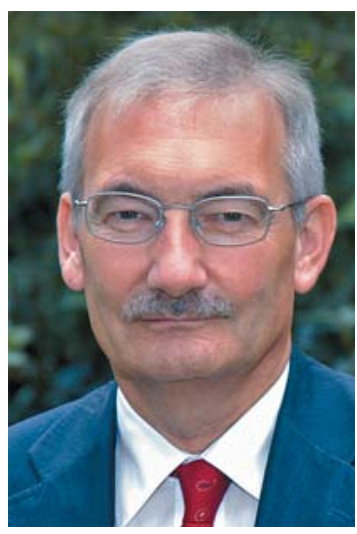

G. Ertl

Bibliografie

DOI 10.1055/s-0031-1272500 Dtsch Med Wochenschr 2011; 136: 167 . (c) Georg Thieme Verlag KG Stuttgart · New York . ISSN 0012-0472

Korrespondenz

Prof. Dr. Georg Ertl

Medizinische Klinik I

Klinikum der Julius-MaximiliansUniversität Josef-Schneider-Str. 2 97080 Würzburg 